\title{
Head and neck cancer treatment with sentinel node biopsy without eletive neck dissection
}

\begin{abstract}
Introduction: The elective neck treatment in patients with squamous cell carcinoma of head and neck is suitable when the neck is clinically negative and the probability of lymphatic metastasis is greater than $20 \%$. However, with this criterion, up to $80 \%$ of surgical specimens will be histopathologically negative. The absence of a method that can determine which patient really presents lymphatic metastasis does not allow us not to perform elective neck dissection when there is high risk for lymphatic metastasis. The sentinel lymph node biopsy seems to be clinically safe as a staging procedure. Besides, this technique apparently removes all the metastatic lymph nodes, since only $3 \%$ to $5 \%$ of the necks would present lymphatic metastasis other than sentinel node and neck recurrence seems similar to that after elective neck dissection. Then, this technique could be justified even as therapeutically, without elective neck dissection, when this lymph node is histologically positive.
\end{abstract}

Objective: The objective of this study was to use the sentinel node technique with lymphocintigraphy and "gamma probe" in patients with squamous cell carcinoma of head and neck, therapeutically, as selective surgical treatment of neck, in substitution to elective neck dissection in clinically negative necks.

Case: Prospective clinical study was accomplished with 25 patients with squamous cell carcinoma of head and neck, staged as T1 to T3 of oral cavity, orpharynx, larynx and face's skin, from May, 2005 to march, 2007. All of them were $\mathrm{cN} 0$, without previous treatment, with indication of elective neck dissection and radiologically negative at neck CT scan. Sentinel node was located and dissected off with handheld "gamma probe" and 2 was directed for histopathological exam and immuno histochemistry evaluation. In the presence of adverse prognostic factors in the primary tumor or multiple lymphatic metastases, patient was submitted to adjuvant radiation therapy. Subjects were followed after surgery monthly with clinical exam and half-yearly with neck CT scan.

Results: The global cervical recurrence rate was $4 \%(1 / 25)$ and in stage IV patients was $25 \%(1 / 4)$. Seven out of 25 patients $(28 \%)$ were sentinel node positive, six of whom were classified as $\mathrm{N} 1$ and one as N2. The average follow up time was eight months, with a range from one to eighteen months.

Conclusion: The sentinel node biopsy stays as a promising method in the neck treatment in head and neck tumors. However, more follow up time, with more patients, is imperative to achieve significant results to establish the sentinel node biopsy without mandatory neck dissection as routine care of head and neck cancer patients with clinically negative necks.

Keywords: head, neck, sentinel node, squamous cell carcinoma, SSC, lymph node, aerodigestive pathway, HISTO, gamma probe, oropharynx, metastases, palpable lymph node, SLNB, peri-neural invasion, vascular emboli, lymphatic emboli
Volume 3 Issue 2 - 2015

\section{Guilherme Machado de Carvalho,Vanessa Gonçalves Silva, Alexandre Caixeta Guimarães, Carlos Takahiro Chone, Agrício Nubiato Crespo \\ Department of Otolaryngology Head and Neck, UNICAMP (Campinas University), Brazil}

Correspondence: Guilherme M de Carvalho, Department Otolaryngology Head and Neck, University of Campinas, Rua Tessália Vieira de Camargo Cidade Universitária $13083-970$ Campinas, SP Postal Code 6I I I, Brazil,Tel +55 I9 352I-7523, Email guimachadocarvalho@gmail.com

Received: October 20, 20I5 | Published: November 6, 2015
Abbreviations: SCC, squamous cell carcinoma; LN, lymph nodes; SLNB, sentinel lymph node biopsy; GP, gamma probe; OC, oral cavity; OP, oropharynx; HISTO, histopathological examination

\section{Introduction}

Squamous cell carcinoma (SCC) of the head and neck, spread preferably via the lymphatic system to the cervical lymph nodes (LN) and controversy still remains about the best techniques to determine which patients actually have cervical lymph node metastases. ${ }^{1}$ Clinical examination and radiological techniques are relatively inaccurate, with about $30 \%$ false negatives and false positives in the determination of the presence of cervical lymph node metastases. ${ }^{2-10}$ The only accurate method for staging the neck is still histopathological examination of the surgical specimen after a neck dissection. ${ }^{11}$ The upper aerodigestive pathway cancer, surgical treatment for the patient's neck is determined according to their risk for occult metastases. In patients whose tumors possibility of greater than $20 \%$ lymphatic metastasis is required neck dissection, even with no palpable lymph node metastasis, due to the benefits in patient survival in $11 \%$ to $14 \%$. This probability varies according to the clinical stage of the tumor. However, the risk of lymph node metastasis of at least $20 \%$ justifies the neck dissection in all patients with this risk, many of the surgical specimens up to $80 \%$, will be histopathologically negative. In the field of cutaneous 
malignant melanoma, a similar debate has been centered on research and treatment of regional lymph nodes and in this respect, the research technique and sentinel lymph node biopsy (SLNB) is embedded. ${ }^{12}$ In this technique, a radiocolloid is injected around a tumor or at the site of previous excision. This radiopharmaceutical is drained for the first chain of lymph nodes, this called SLN. The use of preoperative lymphoscintigraphy and "gamma probe" (GP) intraoperative help in tracking radioactive SLNB that may contain microscopic tumor deposits. ${ }^{13}$ Using this technique in patients with malignant melanoma, it was found that it is highly unlikely that patients with no palpable lymph node disease and SLN free of metastatic disease have metastatic disease in other lymph node groups. ${ }^{14}$

Patients with SCC (squamous cell carcinoma) of the oral cavity (OC) at the early stages, when they have higher leakage than two millimeters, can have considerable risk for cervical lymph node metastasis, with an option of elective neck dissection. ${ }^{15-17}$ The performance of this cervical surgery, as well as resection of the primary tumor, results in increased surgical time and increased surgical morbidity to the patient, with increased time of hospitalization and exposure to surgical risks such as intraoperative bleeding and need for blood transfusion, injury cervical nerves (as accessory vagus, hypoglossal) and vascular lesions (such as the internal jugular vein and carotid arteries), in addition to the risk of anesthetic procedure. ${ }^{15-17}$ Elective neck dissection may add risks described above to the resection of the primary tumor and may be unnecessary on many occasions when his surgical indication is based on clinical criteria of the primary tumor, as is currently done. The absence of a methodology by which to determine which patient really has lymphatic metastasis does not allow us to leave to perform the neck dissection when there is high risk of lymph node metastasis.

Previous studies have shown that the false negative rate for SLNB for SCC of the head and neck, i.e., presence of lymph node metastasis in other non-sentinel lymph nodes in cases with no metástases with SLN ranged from $0 \%$ to $21 \%$, with an average of $3 \% .{ }^{18-33}$ Previous prospective study conducted at the ENT Head and Neck Surgery, the Nuclear Medicine and the Pathology Departments (Campinas University, São Paulo Brazil - UNICAMP), showed excellent outcomes, with false negative rate of SLN about $5.0 \%$. The accuracy for detection of positive and negative lymph nodes compared to histopathology in this study was $96 \% .{ }^{18-33}$ The sensitivity and specificity of the technique to detect occult metastases in lymph nodes was $82 \%(9 / 11)$ and $100 \%$ (39/39), respectively. Based on previous studies with smaller false negative rates of $20 \%$ (value set as a maximum acceptable risk for not performing neck dissection), power would perform the resection of the primary tumor, especially in the OC mouth tumors at early stages, with removal of the SNL for staging and proceed to neck dissection only when these SNL are pathologically committed by SCC. ${ }^{18-33}$

Thus, one can save conducting elective necks dissection with LN negative to the examination, with considerable reduction of surgical morbidity and costs, with savings of nearly $\$ 1,220.00$ dollars per patient. ${ }^{34}$ Based on previous data held in the study of Campinas, with a false negative rate of $5.0 \%$ (meaning that when the SLN is histopathologically negative, there is risk of $5.0 \%$ of occult neck metastases) it is believed that this risk is acceptable to only follow this neck and not submit it to neck dissection, as indicated up this surgery when the risk of occult metastases is greater than $20 \%$. An interesting point, however, has been rising in the literature and also observed in the Campinas study. Apparently the SLNB method detects nearly all metastatic lymph nodes and thus, carrying only the search and removal of SLN even when they are positive, it appears to be safe. ${ }^{29,30}$ This methodology would be appropriate to require reoperation not the patient with neck dissection if the SLN is positive histopathologically for metastasis. Comparative studies, which was also held elective neck dissection subsequent to SLNB, point out that only $3 \%$ to $5 \%$ of metastatic lymph nodes could be left in the patient when performed only the SLNB technique and these probably would have recurrence..$^{29,30,35}$ Because even after radical neck dissection, in clinically and histologically negative necks, it is observed cervical recurrence rate of $6.7 \%$ in a large series of patients. ${ }^{36,37}$ This study aims to show the cervical recurrence rate in the SLNB technique without additional neck dissection in patients with squamous cell carcinoma (SCC) of head and neck (clinic neck and radiologically negative) and would have elective neck dissection indication.

\section{Case presentation}

Patients with SCC of the oral cavity (OC), oropharynx (OP), larynx and skin of the face, which would require neck dissection as part of surgical treatment, with no palpable lymph node metastasis and untreated neck, were invited into the study. From May 2005 to March 2007, 25 patients were included in the study, twelve being with OC, eight OP, four of the larynx and one of the skin of the face. Informed consent was obtained from all patients in this study and the study was approved by the Ethics Committee of the Institution. All had histopathological confirmation of the SCC. Patients with clinical stage T1 to T3 OC, OP, larynx and face and the patients were classified as three of cT1, eleven cT2 and eleven cT3. All patients underwent previous $\mathrm{CT}$ scans of the neck to be eligible for the study as negative neck. All patients underwent two peri-tumoral injections of 0.2 fitato99m MCI-TC two hours before surgery and lymphoscintigraphy two hours after injection. An activity of $25.6 \mathrm{MBq}$ was injected over the normal mucosa surrounding the tumor and the submucosa in the depth of the tumor aspect, in a volume of about $0.2 \mathrm{ml}$ (Figure 1).

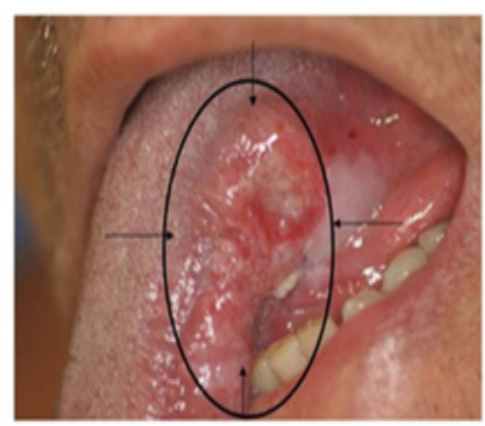

Figure I Example of a tumor on the left side face of the tongue, the black marks indicates the site of peri tumoral injection of drug.

It was taked care to prevent inadvertent leakage of colloid in the mouth. Static images were taken in lateral and anterioposterior projections (Figures 2) (Figure 3) and radioactive lymph nodes were marked with ink in the skin (Figure 4). Next, the primary tumor was resected. With use of GP-1500® Neoprobe manual (Neoprobe Corp., Dublin, OH) (Figure 5) it was located and dissected LS (Figure 6). After the remainder of the neck was reevaluated for the presence of radioactivity and radioactivity confirmed in lymph nodes dissected. Histopathological examination (HISTO) was performed to the SLN with hematoxylin-eosin and was performed immunohistochemical examination of the SLN that were negative to SCC at HISTO examination with hematoxylin-eosin. Next, the patients were followed up on at the clinical office, with semestral clinical examination and cervical CT scan. Patients with sentinel lymph node with extra capsular extension or multiple positive or whose primary tumor present adverse 
factors (involved margins, peri-neural invasion, vascular or lymphatic emboli) underwent postoperative radiotherapy between four and eight weeks of surgery. If only the primary tumor showed adverse factors, postoperative radiotherapy was performed only in the primary tumor bed. If only the sentinel lymph node showed extra capsular extension or there were multiple positive sentinel lymph nodes, postoperative radiotherapy was just in the neck. If the primary tumor had an adverse factor and the sentinel lymph node showed extra capsular extension or multiple positive sentinel lymph nodes, postoperative radiation therapy was the primary and neck.

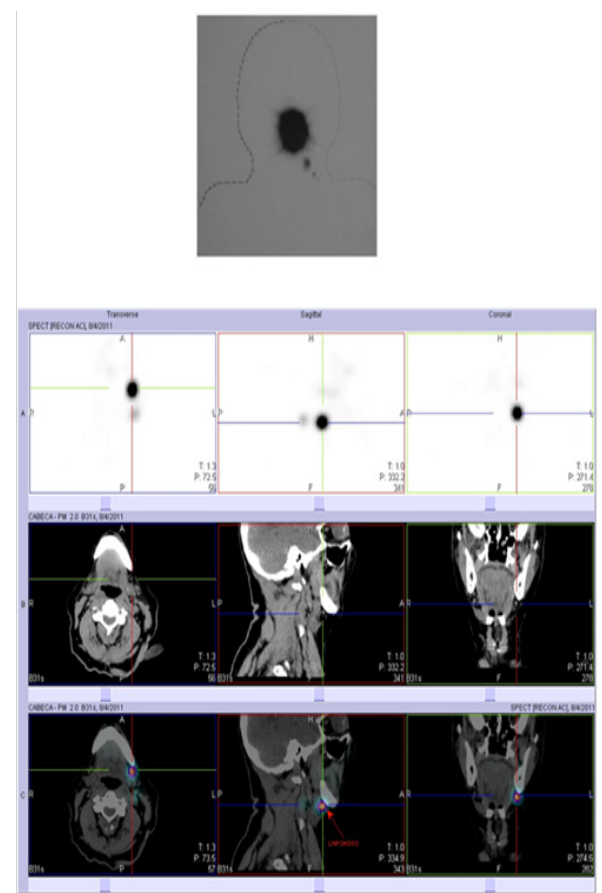

Figure 2 Scintigraphy and SPECT - 30 minutes after peri tumoral injection of drug.
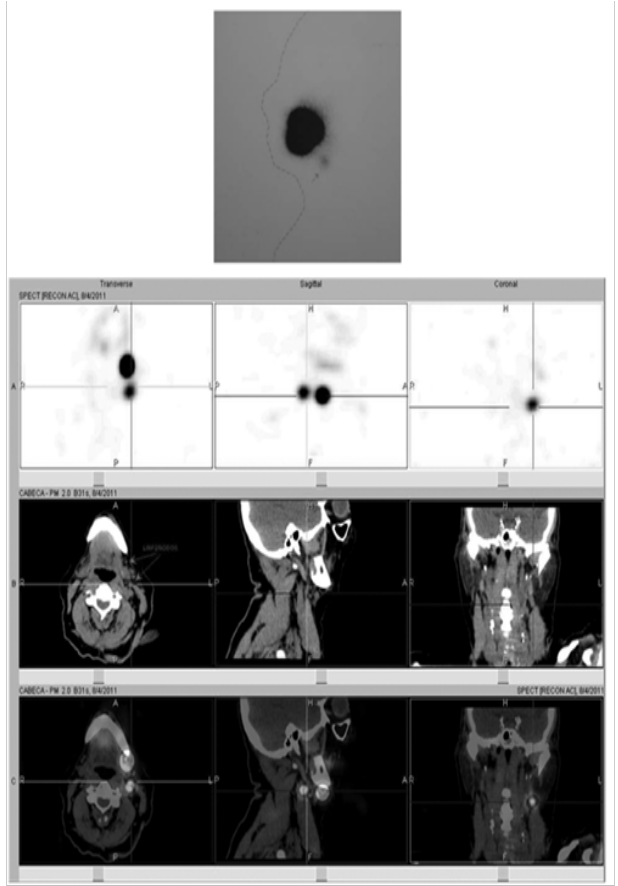

Figure 3 Scintigraphy and SPECT - 30 minutes after peri tumoral injection of drug.

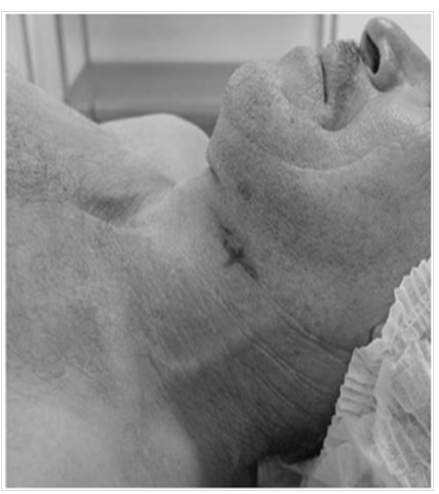

Figure 4 Probable position of the lymph node is marked on the skin (based on scintigraphy and SPECT).
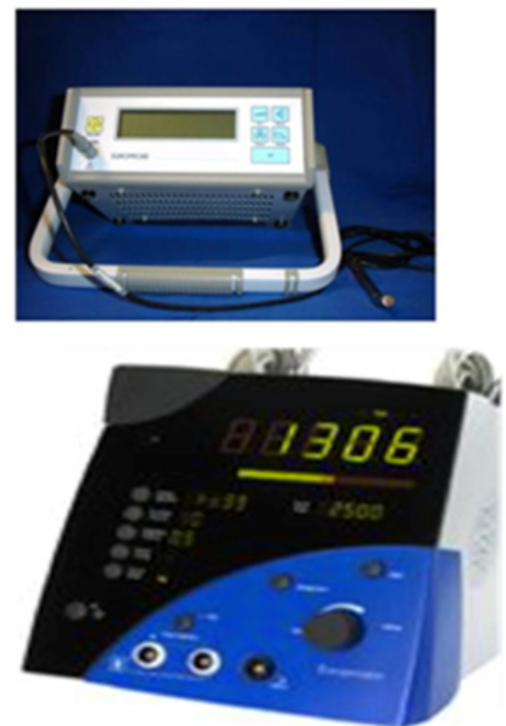

Figure 5 Radiation probe Neoprobe gamma-I500® manual (Neoprobe Corp, Dublin, OH).
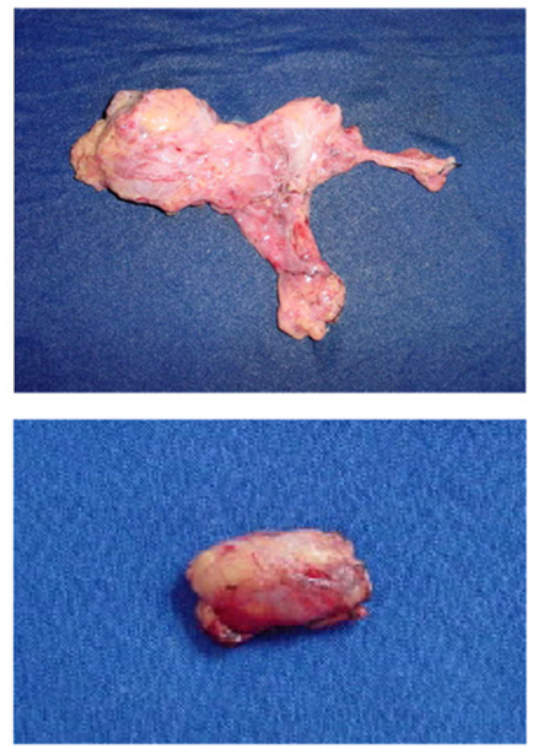

Figure 6 SLNB dissected.

\section{Results}

The post operative follow-up period ranging from one month to 
eighteen months, averaging eight months. The overall neck recurrence rate was $4 \%(1 / 25)$. The categorized relapse rate by pathological staging is itemized in the Table 1 . The SLN dissection included 6 lymph nodes in average $(\min 1-\max 24)$ per patient. Seven patients $(28 \%)$ had SLN positive to SCC, six classified as N1 and one as N2. Fourteen patients were referred for adjuvant radiotherapy, either by adverse factors of the primary tumor or multiple affected sentinel lymph nodes.

Table I Cervical recurrence regarding to the pathological staging

\begin{tabular}{ll}
\hline Stage & $(\%)$ \\
\hline I & $0 / 4(0)$ \\
II & $0 / 10(0)$ \\
III & $0 / 7(0)$ \\
IV & I/4 (25) \\
\hline
\end{tabular}

\section{Discussion}

The SLNB in patients with SCC head and neck was initially used for supraglottis SCC. ${ }^{13}$ By using a combination of preoperative lymphoscintigraphy with intraoperative identification of radioactive lymph nodes with the GP, the results of this study suggest that SLNB method proposed in clinically negative neck is promising in identifying occult metastasis. The global cervical recurrence rates of $4 \%$ and $25 \%$ of stage IV are as expected when compared to recurrence rates in patients undergoing conventional treatment in case the elective neck dissection. Byers and Medina found an overall cervical recurrence rate of $9 \%$ and in stage IV of $18 \%$ in 234 patients with oral cavity and oropharynx tumors, with clinically negative neck, submitted to elective neck dissection..$^{38}$ The cervical recurrence rates in this study are due to the recurrence observed in a patient who presented five sentinel lymph nodes compromised by SCC. This patient was referred to perform adjuvant radiotherapy but initiated the procedure four months after surgery. Several studies have found that a delay in the onset of postoperative radiotherapy is related to an increased recurrence rate and, when indicated, radiation therapy should be started within six weeks after surgery. ${ }^{39-42}$

According to a study which was conducted meta-analysis of SLNB of the head and neck, their use would be safe as staging method. ${ }^{43}$ Due to the small rate of false negative power would perform resection of the primary tumor, especially in the early tumors of the OC, with removal of the SLN for staging and proceed to elective neck dissection only when these SLN are pathologically committed by SCC. Thus, one can save performing elective neck dissection patients with negative SLN to the test, with substantially reduced surgical morbidity and costs. More recent studies indicate that the research method and sentinel lymph node biopsy detects virtually all metastatic lymph nodes, when all the hot lymph nodes are removed and thus creating only the search and removal of the sentinel lymph nodes, even when they are positive, It appears to be safe. ${ }^{29,30}$ This methodology would be appropriate not to require reoperation patients with neck dissection if the sentinel node is positive histopathologically for metastasis.

Thus, based on the concept of operating a clinically negative neck only when the risk of occult metastases is greater than $20 \%$, perhaps the removal of only the sentinel nodes, even if they are positive histopathologically appropriate, since so far, after procedure, the risk of cervical recurrence would be only $3 \%$ to $5 \%,,^{29,30,35}$ this rate lower than that observed in recurrence post radical elective neck dissection. ${ }^{36,44,45}$ It was observed in this study, despite the small segment, the SLNB technique appears to be safe. Patients will be followed rigorously in standard protocol described above and data will be re-evaluated prospectively. In a previous study with 35 patients in our institution, it was observed that there were nine patients with SLN positive Histopathologically and in these patients, all metastases are removed with the SLNB technique, after removing all SLN iodine-accumulating and subsequent elective neck dissection, carried out after removal of the SLN were also all free of metastases, similarly to previous studies. ${ }^{29,30}$ This study proposes a new approach in the treatment of clinically negative neck in patients with head and neck cancer. The SLNB adds no risk or harm to the subjects when compared the risks that would be exposed if subjected to conventional treatment in case the neck dissection. Moreover, it is a study of great social impact, due to the high prevalence of head and neck cancer in the population. The SCC of the oral cavity is the most prevalent in the head and neck are, ranks fifth among the most prevalent cancers in Brazilian male population.

\section{Conclusion}

The sentinel lymph node biopsy is a promising to the neck treatment in patients with SCC of the head and neck. There were $28 \%$ of patients $(7 / 25)$ with lymph node metastases in SLNB in this study and the overall rate of cervical recurrence was $4 \%(1 / 25)$. A longer follow-up, with a larger number of patients is necessary to achieve conclusive and significant results that could consolidate the method as selective neck treatment option in cases of SCC of the head and neck.

\section{Acknowledgements}

None.

\section{Conflict of interest}

The author declares no conflict of interest.

\section{References}

1. Shah JP, Andersen PE. The impact of patterns of nodal metastasis on modifications of neck dissection. Ann Surg Oncol. 1994;1(6):521-532.

2. Byers RM. Modified neck dissection:a study of 967 cases from 1970 to 1980. Am J Surg. 1985;150(4):414-421.

3. Shah JP. Patterns of cervical lymph node metastasis from squamous cell carcinoma of the upper aerodigestive tract. Am J Surg. 1990;160(4):405409.

4. O'brien CJ. A selective approach to neck dissection for mucosal squamous cell carcinoma. Aust N Z J Surg. 1993;64(4):236-241.

5. Kowalski LP, Magrin J, Waksman G, et al. Supraomohyoid neck dissection in the treatment of head and neck tumors:survival results in 212 cases. Arch Otolaryngol Head Neck Surg. 1993;119(9):958-963.

6. Atula TS, Grénman R, Varpula MJ, et al. Palpation, ultrasound, and ultrasound guided fine-needle aspiration cytology in the assessment of cervical lymph node status in head and neck cancer patients. Head Neck. 1996;18(6):545-551

7. Braams JW, Pruim J, Freling NJM, et al. Detection of lymph node metastases of squamous cell cancer of the head and neck with FDG-PET and MRI. J Nucl Med. 1995;36(2):211-216.

8. Don DM, Anzai Y, Lufkin RB, et al. Evaluation of cervical lymph node metastases in squamous cell carcinoma of the head and neck. Laryngoscope. 1995;105:669-674. 
9. Mcguirt WF, Williams DW, Keyes Jr JW, et al. A comparative diagnostic study of head and neck nodal metastases using Positron Emission Tomography. Laryngoscope. 1995;105:373-375.

10. Merrit RM, Willians MF, James TH, et al. A meta-analysis comparing computed tomography with physical examination. ArchOtolaryngol Head NeckSurg. 1997;123(2):149-152.

11. Woolgar JA, Beirne JC, Vaughan ED, et al. Correlation of histopathologic findings with clinical and radiologic assessments of cervicallymph node metastases in oral cancer. Int J Oral MaxillofacSurg. 1995;24(pt $1): 30-37$.

12. Morton DL, Wen DR, Wong JH, et al. Technical details of intraoperative lymphatic mapping for early stage melanoma. ArchSurg. 1992;127(4):392-399.

13. Alex JC, Krag DN. Gamma-probe guided localization of lymph nodes. Surg Oncol. 1993;2:137-143.

14. Reintgen D, Cruse CW, Wells K, et al. The orderly pro-gression of melanoma nodal metastases. Ann Surg. 1994;220(6):759-767.

15. Ambrosch P, Kron M, Fischer G, et al. Micrometastases in carcinoma of the upper aerodigestive tract:detection, risk of metastasizing, and prognostic value of depth of invasion. Head Neck. 1995;17(6):473-479.

16. Mohit-Tarabatai MA, Sobel HJ, Rush BF, et al. Relation of thickness of floor of mouth stage I and II cancers to regional metastasis. Am J Surg. 1986;152(4):351-353

17. Spiro RH, Huvos AG, Wong GY, et al. Predictive value of tumor thickness in squamous cell carcinoma confined to tongue and floor of the mouth. Am J Surg. 1986;152(4):345-350.

18. Koch WM, Choti MA, Civelek C, et al. Gamma probe directed biopsy of the sentinel node in oral squamous cell carcinoma. Arch Otolaryngol Head NeckSurg. 1998;124(4):455-459.

19. Shoaib T, Soutar DS, Prosser JE, et al. A suggested method for sentinel node biopsy in squamous cell carcinoma of the head and neck. Head Neck. 1999;21(8):728-733.

20. Stoeckli SJ, Steinert H, Pfaltz M, et al. Is there a role for positron emission tomography with $18 \mathrm{~F}$-fluorodeoxyglucose in the initial staging of nodal negative oral and oropharyngeal squamous cell carcinoma. Head Neck. 2002;24(4):345-349.

21. Werner JA, Dünne AA, Ramaswamy A, et al. The sentinel node concept in head and neck squamous cell carcinoma:a critical analysis in 100 patients. Laryngorhinootologie. 2002;81(1):31-39.

22. Nieuwenhuis EJ, Castelijns JA, Pijpers R, et al. Wait-and-see policy for the N0 neck in early-stage oral and oropharyngeal squamous cell carcinoma using ultrasonography-guided cytology:is there a role for identification of the sentinel node? Head Neck. 2002;24(3):282-289.

23. Altinyollar H, Berberoglu U, Celen O. Lymphatic mapping and sentinel lymph node biopsy in squamous cell carcinoma of the lower lip. Eur $J$ Surg Oncol. 2002;28(1):72-74.

24. Mozzillo N, Chiesa F, Botti G, et al. Sentinel node biopsy in head and neck cancer. Ann Surg Oncol. 2001;8(9Suppl):103S-105S.

25. Dünne AA, Külkens C, Ramaswamy A, et al. Value of sentinel lymphonodectomy in head and neck cancer patients without evidence of lymphogenic metastatic disease. AurisNasus Larynx. 2001;28(4):339-344.

26. Kovács AF, Acker P, Berner U, et al. Sentinel lymph node excision Treatment method of the N0 neck in patients with oral and oropharyngeal carcinoma. HNO. 2001;49(8):646-653.

27. Stoeckli SJ, Steinert H, Pfaltz M, et al. Sentinel lymph node evaluation in squamous cell carcinoma of the head and neck. Otolaryngol Head Neck Surg. 2001;125(3):221-226.
28. Höft S, Maune S, Muhle C, et al. Sentinel lymph-node biopsy in head and neck cancer. British J Cancer. 2004;91(1):124-128.

29. Werner JA, Dünne AA, Ramaswany A, et al. The sentinel node concept in head and neck cancer: solution for the controversies in the N0 neck? Head Neck. 2004;26(7):603-611.

30. Tschopp L, Nuyens M, Stauffer E, et al. The value of frozen section analysis of the sentinel lymph node in clinically N0 squamous cell carcinoma of the oral cavity and oropharynx. Otolaryngol Head Neck Surg. 2005;132(1):99-102

31. Hart RD, Nasser JG, Trites JR, et al. Sentinel lymph node biopsy in N0 squamous cell carcinoma of the oral cavity and oropharynx. Arch Otolaryngol Head Neck Surg. 2005;131(1):34-38.

32. Ross GL, Shoaib T, Soutar DS, et al. The first international conference on sentinel node biopsy in mucosal head and neck cancer and adoption of a multicenter trial protocol. Ann Surg Oncol. 2002;9(4):406-410.

33. Ross GL, Soutar DS, MacDonald DG, et al. Sentinel node biopsy in head and neck cancer:preliminary results of a multicenter trial. Ann Surg Oncol. 2004;11(7):690-696.

34. Kosuda S, Kusano S, Kohno N, et al. Feasibility and cost-effectiveness of sentinel lymph node radiolocalization in stage N0 head and neck cancer. Arch Otolaryngol Head Neck Surg. 2003;129:1105-1109.

35. Kovács AF, Landes CA, Hamscho N, et al. Sentinel node biopsy as staging tool in a multimodality treatment approach to cancer of oral cavity and the oropharynx. Otolaryngol Head Neck Surg. 2005;132(4):570 576.

36. Strong EW. Preoperative radiation and radical neck dissection. Surg Clin North Am. 1969;49:271-276.

37. Colletier PJ, Garden AS, Morrison WH, et al. Postoperative radiation for squamous cell carcinoma metastatic to cervical lymph nodes from an unknown primary site:outcomes and patterns of failure. Head Neck. 1998;20(8):674-681.

38. Medina JE, Byers RM. Supraomohyoid neck dissection: rationale, indications, and surgical technique. Head Neck. 1989;11(2):111-122.

39. Dietl B, Schafer C, Kolbl O. The prognostic value of time parameters in adjuvant radiotherapy of head and neck cancer. A retrospective analysis of 138 patients. Strahlenther Onkol. 2005;181(12):800-809.

40. Huang J, Barbera L, Brouwers M, et al. Does delay in starting treatment affect the outcomes of radiotherapy? A systematic review. J ClinOncol. 2003;21(3):555-563

41. Muriel VP, Tejada MR, de Dios Luna del Castillo. Time-dose-response relationships in postoperatively irradiated patients with head and neck squamous cell carcinomas. Radiother Oncol. 2001;60(2):137-145.

42. Isaacs JH, Stiles WA, Cassisi NJ, et al. Postoperative radiation of open head and neck wounds updated. Head Neck. 1997;19(3):194-199.

43. Paleri V, Rees G, Arullendran P, et al. Sentinel node biopsy in squamous cell cancer of the oral cavity and oral pharynx: a diagnostic meta-analysis. Head Neck. 2005;27:739-747.

44. Spiro RH, Morgan GJ, Strong EW, et al. Supraomohyoid neck dissection. Am J Surg. 1996;172(6):650-653.

45. Chone CT, Silva AR, Altemani A, et al. Regional tumor recurrence after supraomohyoid neck dissection. Arch Otolaryngol Head Neck Surg. 2003;129(1):54-58. 